\title{
Introducing a six sigma process control technique in a food production line: step-by- step guideline and critical elements of the implementation
}

\begin{abstract}
In the era of digitalisation, the measurement, monitoring and controlling of quality processes is vital and considered the critical dimension in the Six Sigma application toward process excellence. Despite the importance of quality in the food processing, there is a dearth of systematic procedure of process control practices in this industry due to the lack of awareness and guidelines available. Therefore, the purpose of this study are to develop step-by-step guidelines to implement Statistical Process Control as one of the Six Sigma approaches for process control, determine the tools appropriate at each step of the implementation and identify the critical elements toward the success of its application in the food industry. The method used was the "action-research (AR)" approach since the researchers were actively involved in the briefing the SPC awareness and implementation. There are six steps of an implementation of an SPC pilot project, where along the implementation, specific tools are appropriate at each stage The SPC application in this paper provides insights into practical implementation experience in a food manufacturing operation, as well as lessons learned. Until now, most research addressed the case study of the application of SPC. However, this study critically develop a guideline of the implementation and determine the critical element of implementing SPC pilot project.
\end{abstract}

Keyword: SPC; Six sigma; Quality improvement; Action research; Production and planning control 